\title{
Computer-aided simulation and testing of spatial linkages with joint mechanical errors
}

\author{
E. Pezzuti $i^{\ddagger}$, R. Stefanelli ${ }^{\S}$, P. P. Valentini* ${ }^{* \dagger}$ and L. Vita ${ }^{\mathbb{I}}$ \\ Department of Mechanical Engineering, University of Rome Tor Vergata, Via del Politecnico, \\ 1-00133, Rome, Italy
}

\begin{abstract}
SUMMARY
Tolerance allocation influences production costs in a big way. For this reason it is very important to have an accurate study about the effects of manufacturing errors on the functioning and performances of linkages. In this paper, the authors present a computer-aided methodology based on a 3D geometrical approach using the dual-algebra fundamentals. The purpose is to give an useful tool which can be integrated into CAD software in order to evaluate the performances of spatial mechanisms with mechanical errors. The proposed methodology has been validated by means of experimental tests on a Cardan joint mechanism with clearances, misalignments and dimensional errors. Copyright (C) 2005 John Wiley \& Sons, Ltd.
\end{abstract}

KEY WORDS: spatial linkages; dual algebra; tolerance allocation; computer-aided simulation

\section{INTRODUCTION}

Tolerance allocation is not a simple task for a designer. On the one hand, very accurate tolerance requirement improves the quality of the manufactured product assuring high performance, but it causes increasing of costs. Inaccurate tolerances, instead, may cause malfunctioning, bad performances or even problems during assembling. The modern CAD software products provide powerful techniques to assemble the entire model starting from single parts. The constraining functions which can be included mimic the ideal joints (i.e. hinges, guides, screws, cams, etc.) between parts. Simulating mechanical errors is not so simple and it requires ad hoc procedure.

\footnotetext{
*Correspondence to: P. P. Valentini, Department of Mechanical Engineering, University of Rome Tor Vergata, Via del Politecnico, 1-00133, Rome, Italy.

†E-mail: valentini@ing.uniroma2.it

*E-mail: pezzuti@mec.uniroma2.it

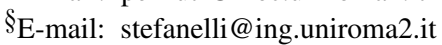

IE-mail: vita@ing.uniroma2.it

Contract/grant sponsor: Ministry for Education, University and Research (MIUR)
}

Received 22 February 2005

Revised 15 July 2005

Copyright (c) 2005 John Wiley \& Sons, Ltd.

Accepted 28 July 2005 
Many researchers proposed linear or non-linear model to study in depth the effects of errors in manufactured mechanisms [1-8]. They are often based on the study of planar mechanisms or assembly chain because many problems can be approximated as $2 \mathrm{D}$ ones. In many cases the design of spatial linkages requires a more accurate study [9-11]. In these cases, simple models are not sufficient to describe the effects of mechanical errors on kinematics and dynamics, and a more complex analysis has to be performed. For this reason the authors present a computer-aided methodology developed using the dual-algebra approach [12]. This numerical methodology [13] is very suitable for describing misalignment and backlash in kinematic pairs [14]. It allows one to write down and implement the kinematic equations in a very short form using only few parameters to describe the variety of manufacturing errors. The proposed methodology has been applied to the analysis of a Cardan joint with manufacturing errors. Recently, a complete dynamic analysis of this mechanical device has been presented in a series of papers authored by Freudenstein and coworkers $[8,11,12,15]$. In the mentioned references, although mounting errors are included, the presence of friction in the kinematic pairs is neglected.

Results from the proposed numerical model can be compared to those from experimental tests. For this reason, the authors build up a computer-aided test rig where several electrical devices and sensors are interlaced to a computer unit in order to simulate joint working conditions and measure speed and torque values affected by mechanical errors.

\section{DUAL-ALGEBRA THEORETICAL BASES FOR KINEMATIC ANALYSIS OF SPATIAL LINKAGES}

In this section, some details about dual algebra [12] will be presented. We can define the dual vector as

$$
\hat{V}=\mathbf{v}+\varepsilon \mathbf{w}
$$

where $\mathbf{v}$ is the geometrical vector, $\varepsilon$ is the dual operator $\left(\varepsilon^{2}=0\right)$ and $\mathbf{w}$ is the moment of vector $\mathbf{v}$ w.r.t. a chosen point. In the same way we can define the dual angle between two axes as

$$
\hat{\theta}=\theta+\varepsilon s
$$

where $\theta$ is the geometrical angle, $\varepsilon$ is the dual operator and $s$ is the minimum distance between the axes. The angle $\theta$ is defined positive if, in the motion to superimpose axis $E_{1}$ on axis $E_{2}$, a counterclockwise ${ }^{\|}$rotation is applied (see Figure 1). The distance $s$ is positive if the axis $E_{1}$ moves towards $E_{2}$ in the direction of $E_{12}$.

For spatial linkage analysis we can rearrange the approach suggested in References [16,17] including dual-algebra entities. For each link (Figure 2) we can define a reference frame $P_{i}-x_{i} y_{i} z_{i}$. A local reference frame $P_{i_{k}}-x_{i_{k}} y_{i_{k}} z_{i_{k}}$ can be assigned to every $k$ th kinematic pair

\footnotetext{
${ }^{\|}$For an observer placed on the side of positive direction of axis $E_{12}$.
} 


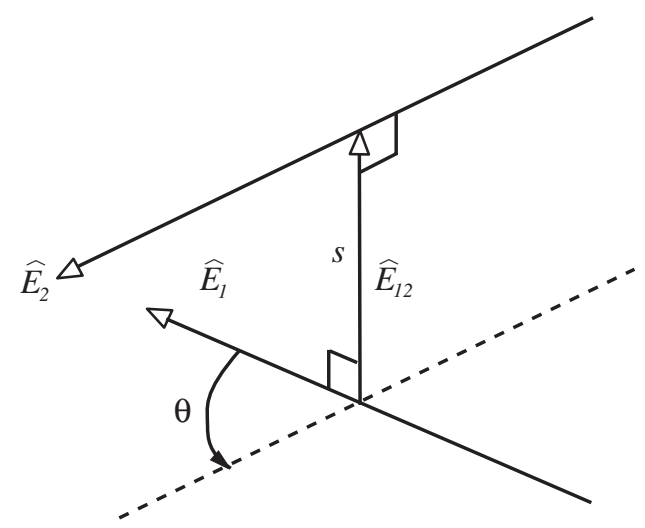

Figure 1. Dual-angle nomenclature.

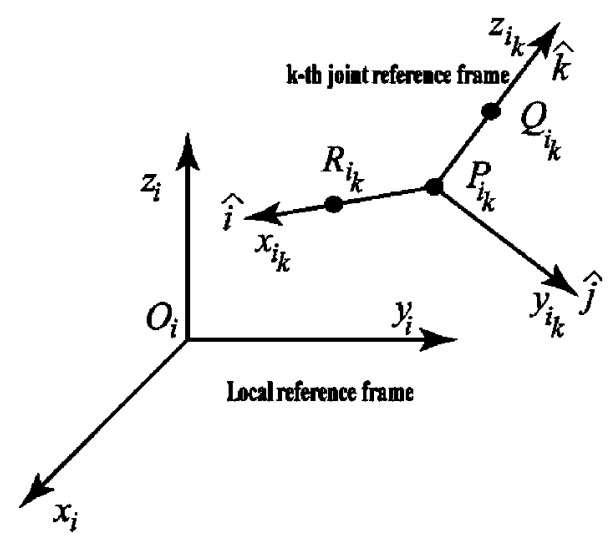

Figure 2. Reference frame nomenclature.

on the $i$ th body. Assuming $P_{i_{k}}$ to be the origin of the reference frame, $R_{i_{k}}$ located at $(1 ; 0 ; 0)$, $Q_{i_{k}}$ located at $(0 ; 1 ; 0)$ we can deduce the dual expression for the unit vector of the reference frame:

$$
\begin{aligned}
& \{\hat{i}\}=\{i\}+\varepsilon[\widetilde{O P}]\{i\} \\
& \{\hat{k}\}=\{k\}+\varepsilon[\widetilde{O P}]\{k\} \\
& \{\hat{j}\}=\left[\begin{array}{ccc}
0 & -\widehat{k_{z}} & \widehat{k_{y}} \\
\widehat{k_{z}} & 0 & -\widehat{k_{x}} \\
-\widehat{k_{y}} & \widehat{k_{x}} & 0
\end{array}\right]\left\{\begin{array}{l}
\widehat{i_{x}} \\
\widehat{i_{y}} \\
\widehat{i_{z}}
\end{array}\right\}
\end{aligned}
$$


where

$$
[\widetilde{O P}]=\left[\begin{array}{ccc}
0 & -P_{z} & P_{y} \\
P_{z} & 0 & -P_{x} \\
-P_{y} & P_{x} & 0
\end{array}\right]
$$

The transform matrix between $i$ th body reference frame and $k$ th joint reference frame on the same body is as follows:

$$
\left.\widehat{A_{i}^{i_{k}}}\right]=\left[\begin{array}{ccc}
\widehat{i_{x}} & \widehat{j_{x}} & \widehat{k_{x}} \\
\widehat{i_{y}} & \widehat{j_{y}} & \widehat{k_{y}} \\
\widehat{i_{z}} & \widehat{j_{z}} & \widehat{k_{z}}
\end{array}\right]
$$

Deducing all the transform matrices, it is possible to write down the closure loop equations for a generic single loop mechanism with $N$ bodies. In fact, for each pair of connected bodies (body $i$ and body $i+1$ ) we express first the transformation between body $i$ and the first $(k=1)$ kinematic pair on the same body (matrix $\left[\widehat{A_{i}^{i_{1}}}\right]$ ), then the transformation between the two reference frames of the two kinematic pairs of body $i$ (matrix $\left[\widehat{A_{i_{1}}^{i_{2}}}\right]$ ). Then, considering the same kinematic pair belonging to body $i$ and body $i+1$ we can define the transform matrix $\widehat{\left[A_{i_{2}}^{(i+1)}\right]}$; finally, we can pass from the joint reference frame of the first kinematic pair on body $i+1$ to the reference frame of body $i+1$ by means of matrix $\left.\widehat{\left[A_{(i+1)}^{(i+1)}\right.}\right]$. Repeating this computation for all the bodies in the kinematic chain we get

$$
\left.\left.\prod_{i=1}^{N}\left[\widehat{A_{i}^{i_{1}}}\right]\left[\widehat{A_{i_{1}}^{i_{2}}}\right] \widehat{\left[A_{i_{2}}^{(i+1)}\right.}\right] \widehat{\left[A_{(i+1)}^{(i+1)}\right.}\right]=[I]
$$

where $i+1=1$ when $i=N$.

\section{PIN-HOLE JOINT WITH ERRORS}

In the classical kinematic model with ideal joints, a pin-hole connection can be described by imposing the coincidence between the origins and $z$ axes of the joint reference frames belonging to the two different bodies connected. Modelling an actual joint is more complex because of clearances and possible misalignments.

Let us assume the pin on the $i$ th body and the hole on the $j$ th body. We can define on the pin a $k$ th local reference frame $P_{i_{k}}-x_{i_{k}} y_{i_{k}} z_{i_{k}}$; in the same way we can define a $k$ th local reference frame $P_{j_{k}}-x_{j_{k}} y_{j_{k}} z_{j_{k}}$ at the hole on the $j$ th body. In the ideal case the $z$ axes of the two reference frames are coincident. In this case, the dual transform matrix between these 
two reference frames is

$$
\left.\widehat{\left[A_{i_{k}}^{j_{k}}\right.}\right]=\left[\begin{array}{ccc}
\cos \widehat{\theta_{i}} & -\sin \widehat{\theta_{i}} & 0 \\
\sin \widehat{\theta_{i}} & \cos \widehat{\theta_{i}} & 0 \\
0 & 0 & 1
\end{array}\right]
$$

where $\widehat{\theta_{i}}=\theta_{i}+\varepsilon s_{i}$ is the dual angle between the unit vectors $\widehat{i_{i_{k}}}$ and $\widehat{i_{j_{k}}}$. Now we can deduce the transform matrix between the $O_{i}-x_{i} y_{i} z_{i}$ reference frame which is placed on $i$ th body and the $O_{j}-x_{j} y_{j} z_{j}$ reference frame placed on the $i$ th body, as

$$
\left.\left.\widehat{\left[A_{i}^{j}\right.}\right]=\left[\widehat{A_{i}^{i_{k}}}\right]\left[\widehat{A_{i_{k}}^{j_{k}}}\right] \widehat{A_{j_{k}}^{j}}\right]
$$

Let us now remove the hypothesis of ideal joint and assume the following:

- Kinematic elements can have two or infinite points of contact.

- When the contact point are two $\left(B_{1}\right.$ and $\left.B_{2}\right)$, these lay on the outer/inner edge of the pin (Figure 3).

- The possible errors of the pair can be radial backlash and cylindricity tolerances.

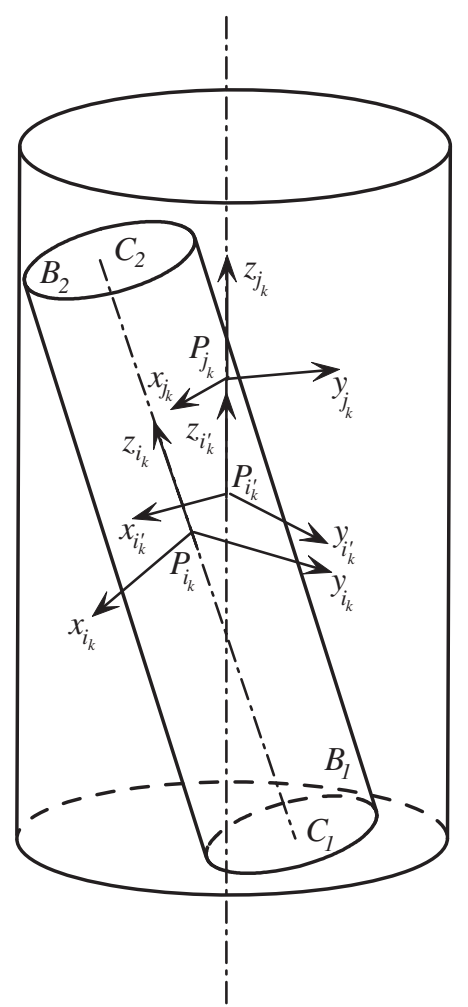

Figure 3. Pin-hole joint with backlashes. 
In order to describe the actual position of the pin inside the hole we have to define another reference frame $P_{i_{k^{\prime}}}-x_{i_{k^{\prime}}} y_{i_{k^{\prime}}} z_{i_{k^{\prime}}}$ with the $z_{i_{k^{\prime}}}$ axis aligned to $z_{j_{k}}$ axis. In the ideal joint this reference frame coincides with $P_{i_{k}}-x_{i_{k}} y_{i_{k}} z_{i_{k}}$; in the presence of error it will be necessary to describe a screw motion in order to move from one frame to the other (see Figure 3).

The goal is to deduce the parameters of this screw motion as a function of the $B_{1}$ and $B_{2}$ contact points location in order to compute $\left[\widehat{A_{i_{k}}^{i_{k^{\prime}}}}\right]$ matrix. Because of the misalignment between the pin and hole axes, Equation (10) has to be rearranged as

$$
\left.\left[\widehat{A_{i}^{j}}\right]=\left[\widehat{A_{i}^{i_{k}}}\right]\left[\widehat{A_{i_{k}}^{i_{k^{\prime}}}}\right]\left[\widehat{A_{i_{k^{\prime}}}^{j_{k}}}\right] \widehat{A_{j_{k}}^{j}}\right]
$$

On the right side of Figure 4 the mid-plane which is orthogonal to the hole axis is depicted. On this plane, we can draw the projection of pin edges and contact points. The projection of the two circular edges should be two ellipses, but for the sake of simplicity we assume them to be two circles. This assumption is justified by the smallness of the possible clearance and misalignment error.

With reference to Figure $4, C_{1}^{\prime}$ and $C_{2}^{\prime}$ are the projections of the circular edge centres, $B_{1}^{\prime}$ and $B_{2}^{\prime}$ are the projections of two contact points. Moreover, $\beta_{i j}$ is the angle between the $B_{1}^{\prime} P_{i_{k}}$ axis and $B_{2}^{\prime} P_{i_{k}}$ axis and $\xi_{i j}$ is the angle between $P_{i_{k}} P_{i_{k^{\prime}}}$ axis and $x_{i_{k^{\prime}}}$ axis. These two parameters $\left(\beta_{i j}\right.$ and $\left.\xi_{i j}\right)$ are sufficient to completely describe the position of the pin inside the hole.
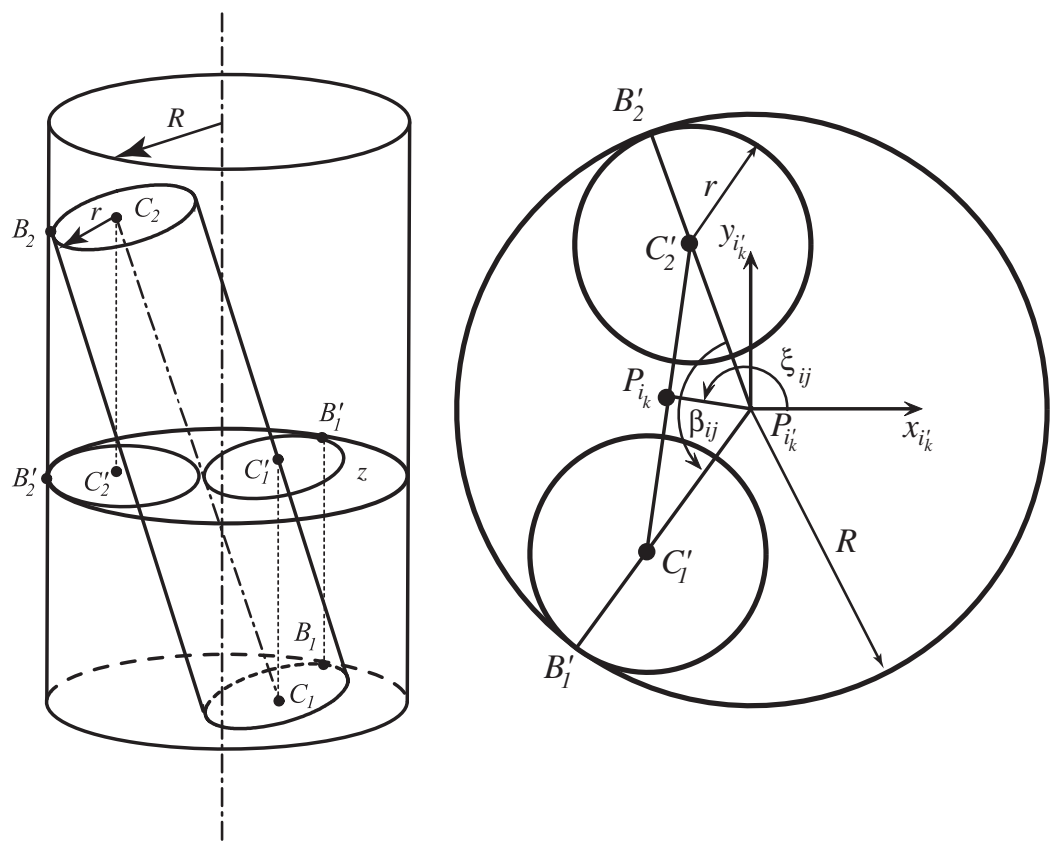

Figure 4. Pin-hole joint nomenclature. 
The co-ordinates of $C_{1}$ and $C_{2}$ in the reference frame $P_{i_{k^{\prime}}} x_{i_{k^{\prime}}} y_{i_{k^{\prime}}} z_{i_{k^{\prime}}}$ can be expressed as

$$
\begin{aligned}
& x_{C_{1}}=x_{C_{1}^{\prime}} \approx(R-r) \cos \left(\xi_{i j}+\frac{\beta_{i j}}{2}\right) \\
& y_{C_{1}}=y_{C_{1}^{\prime}} \approx(R-r) \sin \left(\xi_{i j}+\frac{\beta_{i j}}{2}\right) \\
& z_{C_{1}}=-\frac{H}{2}
\end{aligned}
$$

and

$$
\begin{aligned}
& x_{C_{2}}=x_{C_{2}^{\prime}} \approx(R-r) \cos \left(\xi_{i j}-\frac{\beta_{i j}}{2}\right) \\
& y_{C_{2}}=y_{C_{2}^{\prime}} \approx(R-r) \sin \left(\xi_{i j}-\frac{\beta_{i j}}{2}\right) \\
& z_{C_{2}}=\frac{H}{2}
\end{aligned}
$$

Then, we can define the following dual vector:

$$
\widehat{C_{1} C_{2}}=\mathbf{C}_{1} \mathbf{C}_{2}+\varepsilon \mathbf{P}_{i_{k^{\prime}}} \mathbf{C}_{1} \times \mathbf{C}_{1} \mathbf{C}_{2}
$$

where $\mathbf{C}_{1} \mathbf{C}_{2}=\left\{\begin{array}{lll}x_{C_{2}}-x_{C_{1}} & y_{C_{2}}-y_{C_{1}} & z_{C_{2}}-z_{C_{1}}\end{array}\right\}^{\mathrm{T}}$ and $H$ is the length of the pin. Equation (14) can be used to build the $\left[\widehat{A_{i_{k}}^{i_{k^{\prime}}}}\right]$ matrix. The details of this derivation can be found in Reference [13]. The closure equations (8) solve the kinematic problem of the mechanism.

\section{MODELLING OF FRICTION}

Under ideal working conditions, the resultant of the reaction forces at the kinematic pairs is null. This is not necessarily true in the presence of radial clearance or manufacturing tolerances. If the presence of friction is considered, then frictional forces (moments) arise along (about) the axis of the kinematic pair $[18,19]$. In order to completely model the effects of friction it is necessary to define the geometric features of the journal bearing.

\subsection{Revolute joint}

The frictional forces are mainly caused by the reaction forces $\left(F_{x_{i}}, F_{y_{i}}, F_{z_{i}}\right)$ and the reaction moments $\left(M_{x_{i}}, M_{y_{i}}\right)$ at the kinematic pair. The effect of $F_{z_{i}}$ could be neglected considering that there is no sliding velocity along the axis of the revolute joint. In particular, the effect of reaction moments could be taken into account considering the equivalent couple of 
forces (Figure 5):

$$
F=\frac{M_{x_{1}}}{L_{i}}
$$

where $F$ is orthogonal to the direction of the axis of the revolute joint.

Thus, the frictional torque due to the reaction moment $M_{x_{i}}$ could be evaluated as follows:

$$
\tau_{f}^{\chi_{i}}=f \frac{M_{x_{i}}}{L_{i}} d_{i}
$$

where $f$ is the friction coefficient, $d_{i}$ is the diameter of the journal bearing and $L_{i}$ is the length of the bearing. In the same way, the frictional torque due to $M_{y_{i}}$ can be evaluated. Therefore, the whole frictional torque about the axis of the journal bearing is

$$
M_{z_{i}}=-\operatorname{sign}\left(\dot{\theta}_{i}\right)\left(\tau_{f}^{(i)}+\frac{f d_{i}}{2} \sqrt{F_{x_{i}}^{2}+F_{y_{i}}^{2}}\right)
$$

where $\dot{\theta}_{i}$ is the relative angular speed of the journal w.r.t. the bearing and

$$
\tau_{f}^{(i)}=\sqrt{\left(\tau_{f}^{x_{i}}\right)^{2}+\left(\tau_{f}^{y_{i}}\right)^{2}}
$$

\subsection{Cylindrical joint}

Following the same methodology illustrated for the revolute joint, the frictional moment can be evaluated by means of (17). In this case, a frictional force along the axis of the kinematic pair arises as well. Its value is defined by the following equation:

$$
F_{z_{i}}=-\operatorname{sign}\left(\dot{s}_{i}\right) f\left(\sqrt{F_{x_{i}}^{2}+F_{y_{i}}^{2}}+2 \frac{\sqrt{M_{x_{i}}^{2}+M_{y_{i}}^{2}}}{L_{i}}\right)
$$

where $\dot{s}_{i}$ is the sliding velocity along the axis of the kinematic pair.
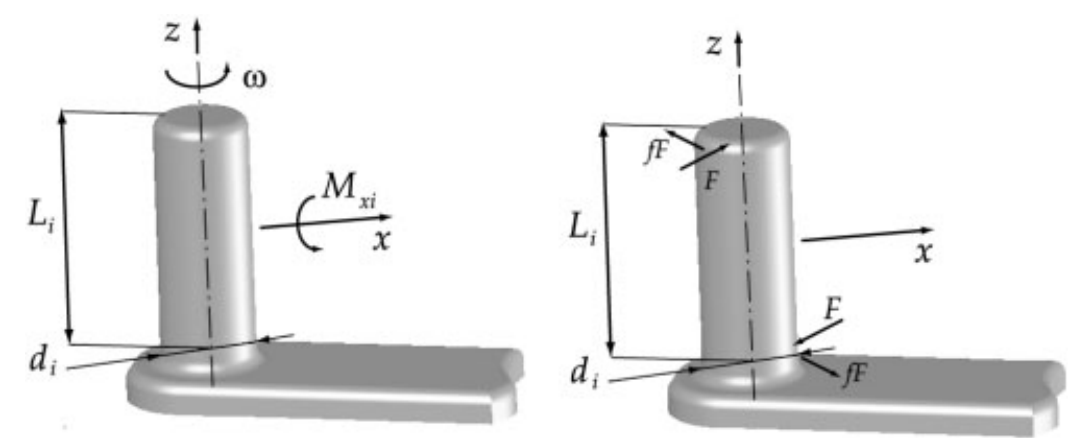

Figure 5. Modelling of friction in kinematic pairs. 


\section{MECHANISM DYNAMICS}

The equation of dynamics is deduced by the derivation of dual-momentum equation. The dual momentum is a dual entity including both momentum and angular momentum. For the generic $i$ th link it can be expressed as

$$
\left\{\hat{H}_{C(i)}\right\}^{\left(i_{k}\right)}=m_{i}\left\{v_{C(i)}\right\}^{\left(i_{k}\right)}-m_{i}\left[\tilde{R}^{\left(i_{k}\right)}\right]\left\{\omega_{i}\right\}^{\left(i_{k}\right)}+\varepsilon\left(m_{i}\left[\tilde{R}^{\left(i_{k}\right)}\right]\left\{v_{C(i)}\right\}^{\left(i_{k}\right)}+\left[J_{C(i)}^{\left(i_{k}\right)}\right]\left\{\omega_{i}\right\}^{\left(i_{k}\right)}\right)
$$

where the real part represents the momentum and the dual part the angular momentum w.r.t. a point $C(i)$. In particular, $\left\{v_{C(i)}\right\}^{\left(i_{k}\right)}$ represents the velocity of body $i$ evaluated in $C$ expressed in reference $i_{k}$; where $i_{k}$ is the $k$ th reference frame on the $i$ th body; $\left\{\omega_{i}\right\}^{\left(i_{k}\right)}$ is the angular velocity of body $i$ in reference $i_{k}$ components; $\left[\tilde{R}^{\left(i_{k}\right)}\right]$ is the skew matrix of vector $R$, which represents the distance between point $C$ and the centre of mass of body $i$ in reference $i_{k}$ components; $\left[J_{C(i)}^{\left(i_{k}\right)}\right]$ is the inertia matrix of body $i$ in $C-x_{i_{k}} y_{i_{k}} z_{i_{k}}$ reference frame and $m_{i}$ is the mass of body $i$.

The equations of motion can be deduced as follows:

$$
\frac{\mathrm{d}}{\mathrm{d} t}\left\{\hat{H}_{C(i)}\right\}^{\left(i_{k}\right)}=\left\{\hat{F}_{C(i)}\right\}^{\left(i_{k}\right)}
$$

where $\left\{\hat{F}_{C(i)}\right\}^{\left(i_{k}\right)}$ is the dual vector of external forces and include both forces and moments evaluated in $C$ and expressed in $i_{k}$ reference frame co-ordinates. Details about the computation of (20) and (21) can be found in Reference [10].

In every pin hole joint, the effect of friction has also been included. This contribution causes some resistant torque and force which can be computed following the same procedure discussed in Reference [10].

\section{NUMERICAL SIMULATION}

In order to give an example of the proposed methodology a Cardan joint has been investigated. The joint, depicted in Figure 6 (on the left side) has been considered as a RCCC mechanism

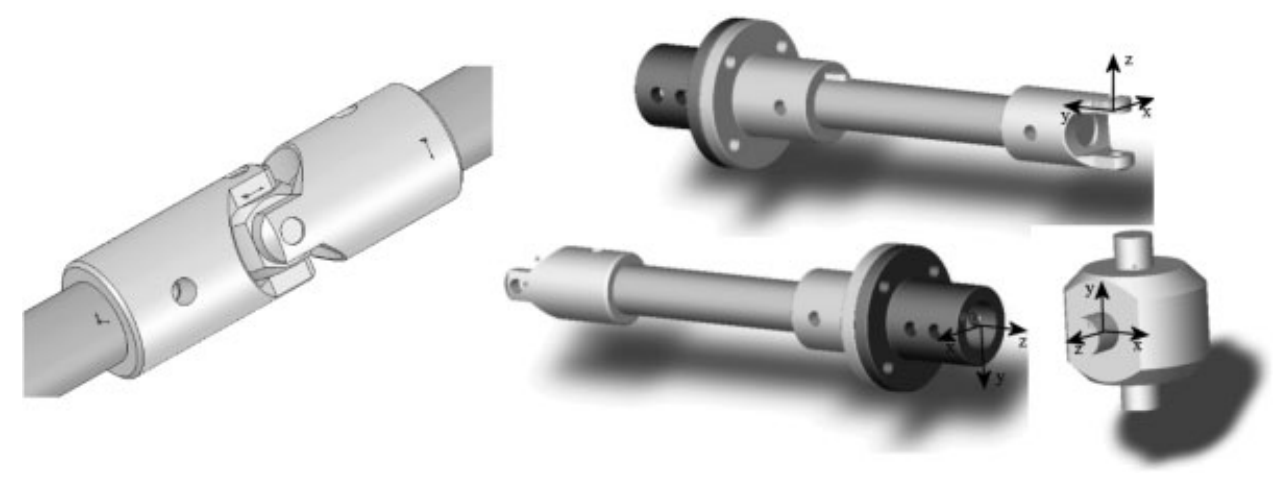

Figure 6. CAD model of the joint and the two shafts. 
made up of three links with their local reference frame located as shown on the right side of the Figure 6.

The first link is an assembly of a flange, input shaft and one end of the Cardan joint; the second link is the central cross and the third one is an assembly of the other end of the joint, the output shaft and a flange. Mass properties of each link are summarized in Table I.

In Table II the geometrical properties and mechanical errors are summarized. Note that $\alpha_{i}$ is the angle between the axes of the two joints on the $i$ th link (Figure 7); $a_{i}$ is the distance between the same axes (this distance is zero if the axes are incident); $R$ is the

Table I. Mass properties of the Cardan joint.

\begin{tabular}{|c|c|c|c|c|c|c|}
\hline Link & $\begin{array}{c}\text { Mass } \\
(\mathrm{kg})\end{array}$ & \multicolumn{4}{|c|}{$\begin{array}{l}\text { Inertia matrix w.r.t. local reference } \\
\text { frame (Figure 4) }\left(\mathrm{kg} \mathrm{m}^{2}\right)\end{array}$} & $\begin{array}{l}\text { Location of centre of mass w.r.t. local } \\
\text { reference frame (Figure } 4)(\mathrm{m})\end{array}$ \\
\hline \multirow{3}{*}{$\begin{array}{l}\text { Input } \\
\text { shaft }\end{array}$} & \multirow{3}{*}{0.936} & \multirow{3}{*}[J]{$=$} & 0.019254 & 0 & 0 & \multirow{3}{*}{$G \equiv(0,0.1277,-0.0086)$} \\
\hline & & & 0 & 0.000252 & -0.001026 & \\
\hline & & & 0 & -0.001026 & 0.019182 & \\
\hline \multirow{3}{*}{$\begin{array}{l}\text { Central } \\
\text { joint }\end{array}$} & \multirow{3}{*}{0.045} & \multirow{3}{*}[J]{$=$} & {$[0.000006$} & 0 & 0 & \multirow{3}{*}{$G \equiv(0,0,-0.0085)$} \\
\hline & & & 0 & 0.000006 & 0 & \\
\hline & & & 0 & 0 & 0.000002 & \\
\hline \multirow{3}{*}{$\begin{array}{l}\text { Output } \\
\text { shaft }\end{array}$} & \multirow{3}{*}{0.936} & \multirow{3}{*}[J]{$=$} & {$[0.012147$} & 0 & 0 & \multirow{3}{*}{$G \equiv(0,0,-0.093782)$} \\
\hline & & & 0 & 0.012148 & 0 & \\
\hline & & & 0 & 0 & 0.000184 & \\
\hline
\end{tabular}

Table II. Kinematic pairs parameters.

\begin{tabular}{lccc}
\hline Kin. pair & $\alpha_{i}$ & $a_{i}(\mathrm{~mm})$ & $R_{i}(\mathrm{~mm})$ \\
\hline 1 & $90-0.1^{\circ}$ & 0.01 & $10+0.01$ \\
2 & $90-0.1^{\circ}$ & 0.01 & $10+0.02$ \\
3 & $90-0.1^{\circ}$ & 0.02 & $10+0.01$ \\
4 & $135-0.1^{\circ}$ & 0.01 & $10+0.02$ \\
$H(\mathrm{~mm})$ & $\Delta r(\mathrm{~mm})$ & $\beta_{i j}$ & $\xi_{i j}$ \\
10 & 0.01 & $0-360^{\circ}$ & $0-360^{\circ}$ \\
\hline
\end{tabular}




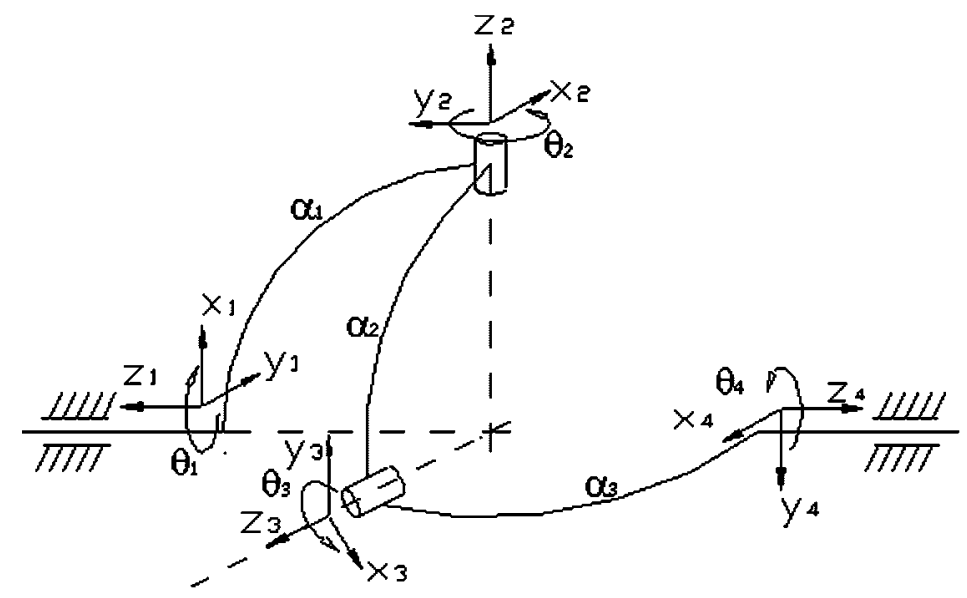

Figure 7. Angles between kinematic pairs.

radius of the pin; $\Delta r$ is the radial clearance and $H$ is the length of the pin, $\beta_{i j}$ and $\xi_{i j}$ are the angles describing pin centre position which are depicted in Figure 4 (on the right). The dynamic coefficient of friction is set equal to 0.42 for each kinematic pair.

\section{EXPERIMENTAL TESTS}

In order to validate and improve the numerical algorithm an experimental test rig has been built (Figure 8). The test rig is made of an electric motor, an electro magnetic brake, two torque/speed sensors and a computer unit which control the devices and acquire the simulation data.

Acting on motor virtual control panel and choosing the desired resisting torque exerted by the brake it is possible to simulate different working scenarios. Moreover, it is possible to simulate angular misalignment of the investigated joint tilting one end of the test rig.

The test rig is equipped with the following instruments:

- An adjustable steel table.

- Two torque/speed transducers (model Magtrol TMB 210 with max torque: $100.00 \mathrm{Nm}$; max speed: 4000 r.p.m.; torque sensitivity $100 \mathrm{mV} / \mathrm{Nm}$; speed sensitivity: 60 pulses per rev.).

- One brushless motor (two poles; peak torque: $110 \mathrm{Nm}$ ) with a control panel and control software.

- One electromagnetic brake (model Merobel SA FRAT 650; max torque 65 Nm; min torque $0.63 \mathrm{Nm}$ ) with a radial fan and a DGT 200 MC digital controller.

- One personal computer with an a/d converter and a National Instrument multichannel acquiring system. 


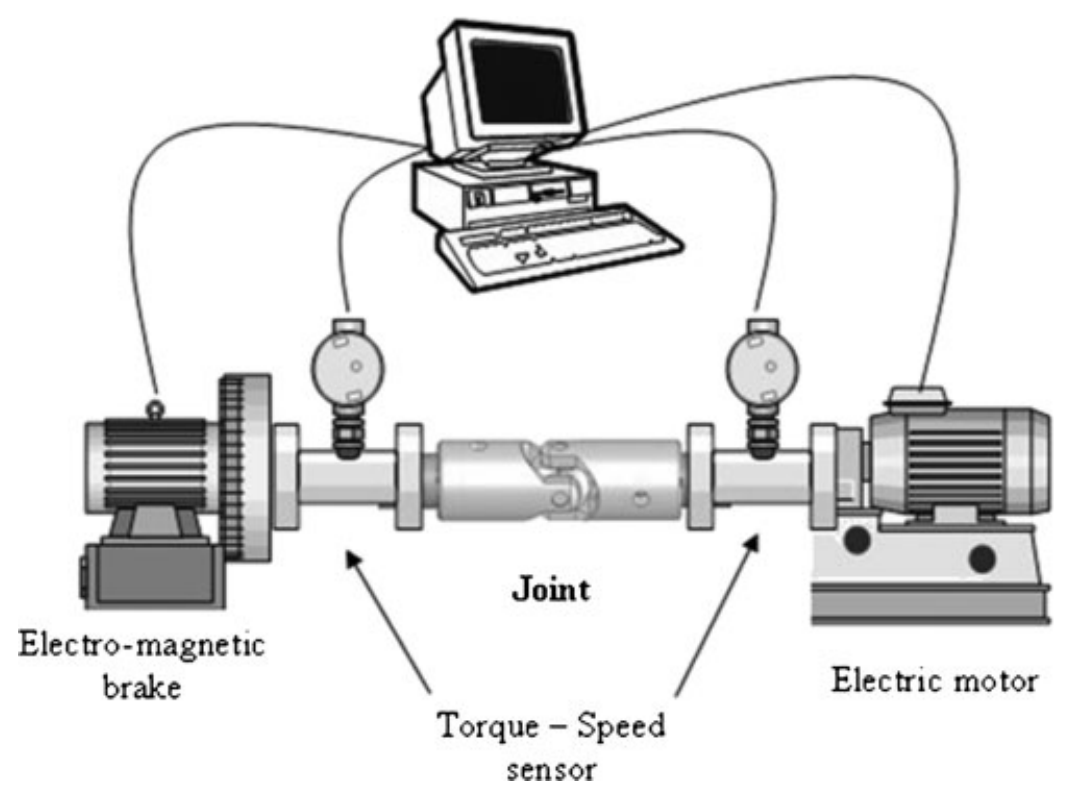

Figure 8. Experimental set-up.

\section{RESULTS AND DISCUSSION}

The investigated Cardan joint has been simulated and tested under several working conditions. The study involved the following working parameters:

- Angular speed of input shaft.

- Angular misalignment between input and output shaft.

- Resisting torque.

The following variables have been measured:

- Torque at input and output shaft.

- Angular speed of input and output shaft.

These variables allow one to compute the efficiency $(\eta)$ of the transmission in the following way:

$$
\eta=\frac{P_{\mathrm{out}}}{P_{\mathrm{in}}}=\frac{T_{\mathrm{out}} \omega_{\mathrm{out}}}{T_{\mathrm{in}} \omega_{\mathrm{in}}}
$$

where $P_{\text {in }}$ and $P_{\text {out }}$ are input and output powers, respectively; $T_{\text {in }}$ and $T_{\text {out }}$ are input and output torques, respectively; $\omega_{\text {in }}$ and $\omega_{\text {out }}$ are input and output angular speeds, respectively.

In Figure 9, the influence of angular misalignment between input and output shafts and the influence of input angular speed on the efficiency have been plotted. It can be noted that if the misalignment between the shafts increases, then the power losses increase too; if the input speed increases, then the power losses increase too. It can be noted that the increasing of 

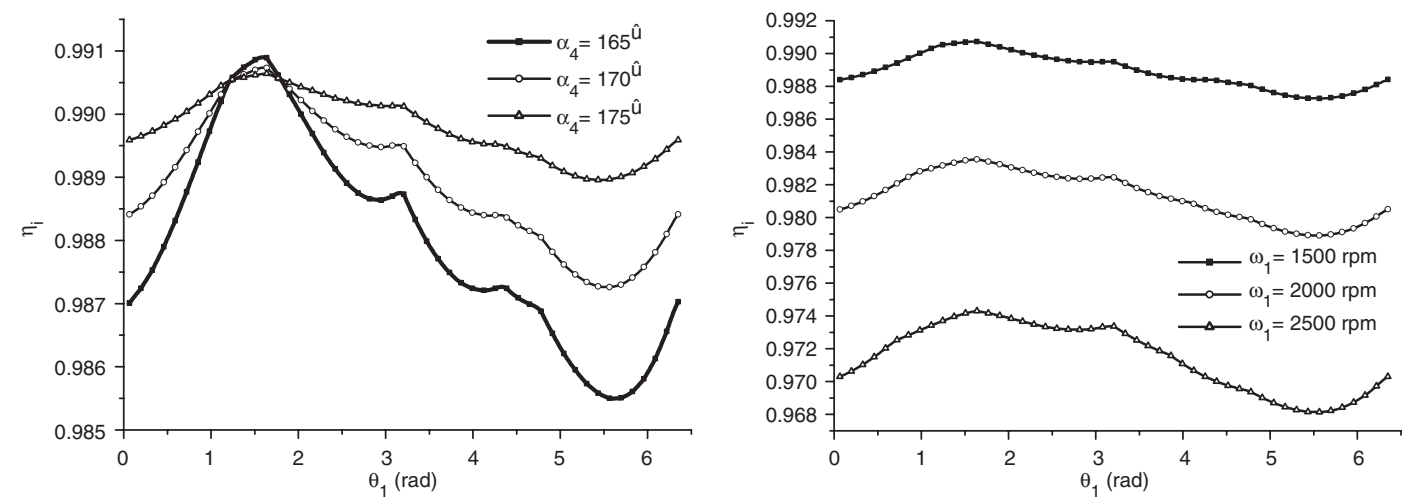

Figure 9. Influence of angular misalignment between input and output shafts on efficiency (on the left); influence of input angular speed on efficiency (on the right).

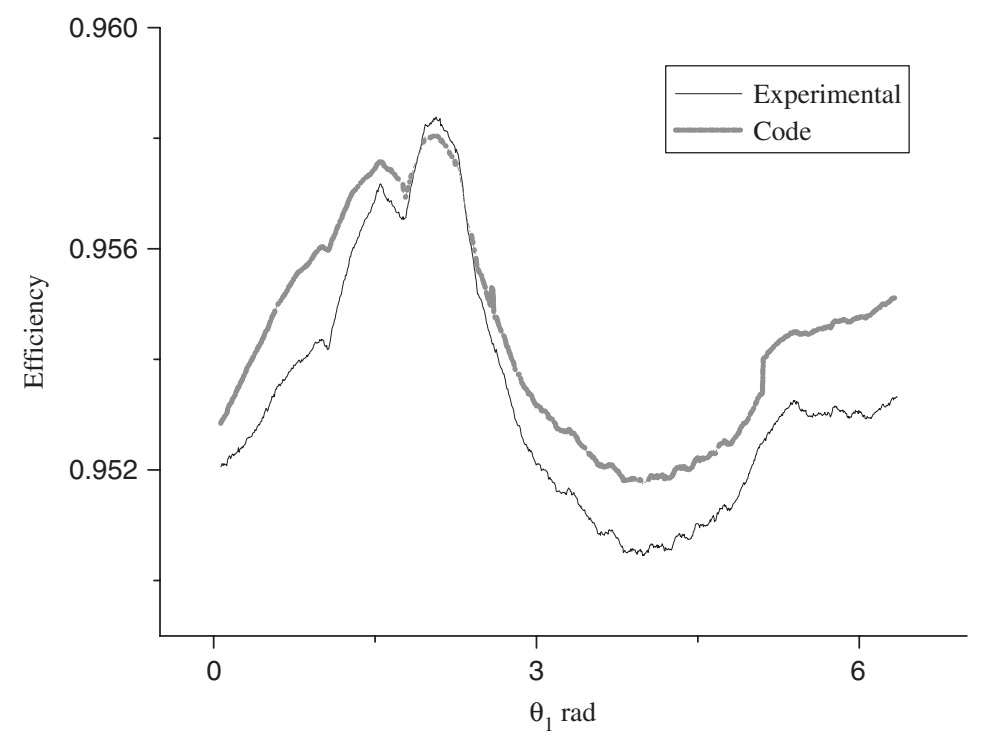

Figure 10. Comparison between measured and simulated efficiency.

misalignment or the increasing of angular speed causes the inertial forces to be higher and so the reaction forces exerted by the joints get higher, and the friction forces increase too.

In Figure 10, a comparison between simulated and experimental efficiency has been made. A very good agreement between the two plots is observed.

\section{CONCLUSIONS}

The proposed computer-aided methodology turned out to be a valid instrument in order to improve the design of spatial linkages. Moreover, the dual-algebra approach seems to be 
convenient in order to easily introduce error in joints. The only disadvantage is the need for the definition of some basic operation between dual entities. This mathematical effort has to be performed only once, then the derivation of both kinematics and dynamics equations is similar to those in the classical multibody approach. The proposed methodology has been applied to an industrial case of an automotive transmission by means of a Cardan joint. The comparison between the numerical results and those from the acquisition using a test rig confirms the validity of the proposed model. Future work will concern the modelling of different types of joints and the transient analysis investigation.

\section{ACKNOWLEDGEMENTS}

The authors wish to acknowledge the grant by Ministry for Education, University and Research (MIUR).

\section{REFERENCES}

1. Sacks E, Joskowicz L. Parametric kinematic tolerance analysis of general planar systems. Computer-Aided Design 1998; 30(9):707-714.

2. Chase KW. Tolerance allocation methods. ADCATS Report No. 99-6, 2000.

3. Chun Zhang, Hsu Pin Wang. Robust design of assembly and machining tolerance allocations. IIE Transactions 1998; 30:17-29.

4. Chase KW, Parkinson AR. A survey of research in the application of tolerance analysis to the design of mechanical assemblies. ADCATS Report No. 91-1, 2000.

5. Forouraghi B. Worst-case tolerance design and quality assurance via genetic algorithms. Journal of Optimization Theory and Applications 2002; 113(2):251-268.

6. Wang HHS, Roth B. Position errors due to clearance in journal bearings. Journal of Mechanisms, Transmission and Automation in Design (ASME) 1989; 111:315-320.

7. Pennestrì E, Vita L. Mechanical efficiency analysis of a Cardan joint with manufacturing tolerances. Proceedings RAAD03, 12th International Workshop on Robotics in Alpe-Adria-Danube Region, Cassino, 7-10 May 2003, Paper No. O53RAAD03.

8. Chen CK, Freudenstein F. Dynamic analysis of a universal joint with manufacturing tolerances. Journal of Mechanisms, Transmissions and Automation in Design (ASME) 1986; 108:524-532.

9. Yang AT. Application of quaternion algebra and dual numbers to the analysis of spatial mechanisms. Doctoral Dissertation, Columbia University, New York, 1963.

10. Cavacece M, Pennestrì E, Valentini PP, Vita L. Mechanical efficiency analysis of a Cardan joint. Proceedings of DETC'04-ASME 200428 September-2 October 2004, Salt Lake City, Utah, U.S.A.

11. Fischer I, Freudenstein F. Internal force and moment transmission in a Cardan joint with manufacturing tolerances. Journal of Mechanisms, Transmissions and Automation in Design (ASME) 1984; 106:301-311.

12. Fischer IS. Dual-Number Methods in Kinematics, Statics and Dynamics. CRC Press: Boca Raton, 1998.

13. Cecchini E, Pennestrì E, Stefanelli R, Vita L. A dual number approach to the kinematic analysis of spatial linkages with dimensional and geometric tolerances. Proceedings of DETC'04-ASME 2004 28 September-2 October 2004, Salt Lake City, Utah, U.S.A.

14. Beyer R. Technische Raumkinematik. Springer: Berlin, 1963.

15. Freudenstein F, Macey JP. The inertia torques of the Hooke joint. Proceedings of the 21st Biennial ASME Mechanisms Conference, Chicago, DE-vol. 24, 16-19 September 1990; 407-413.

16. Nikravesh P. Computer-Aided Analysis of Mechanical Systems. Prentice-Hall: New York, 1988.

17. Haug EJ. Computer-Aided Kinematics and Dynamics of Mechanical Systems, vol. I. Allyn and Bacon, Newton, MA, 1989; 48-104.

18. Shih CW, Shih MY, Haug EJ. Dynamics of mechanical systems with Coulomb friction, stiction, impact and constraint deletion-III. Mechanism and Machine Theory 1986; 21:417-425.

19. Dhanaraj C, Sharan AM. Efficient modeling of rigid link body dynamic problems with friction. Mechanism and Machine Theory 1995; 30:749-764. 